\title{
Paper
}

\section{Spectrum Analysis of Heart Rate Fluctuations in Hatched and Unhatched Prenatal Chick Embryos}

\author{
KENJI MORIYA ${ }^{*} \quad$ Member, $\quad$ YUYA CHIBA ${ }^{\ddagger}$ Non-member \\ YOSHIKO MARUYAMA ${ }^{\S}$ Non-member
}

(Received February 5, 2018, revised May 21, 2018)

\begin{abstract}
Instantaneous heart rate (IHR) was continuously measured in six hatched embryos and three unhatched embryos. Spectrum analysis of IHR was performed to determine indices of the sympathetic and parasympathetic nervous system activity and a spectrum gradient $\alpha\left(1 / \mathrm{f}^{\alpha}\right)$. Developmental patterns of HR and HR fluctuations (HRFs) in successfully hatched embryos were elucidated. Developmental patterns and/or HRFs of unhatched embryos were different compared with those of normally hatched embryos. Although baseline HR and cyclic mean HR changes in unhatched embryos were similar to those in normally hatched embryos, indices of the autonomic nervous system and the spectrum gradient were distinctly different. These results imply abnormal physiological development of embryos could be speculated using time-series and spectrum analyses. In addition, characteristic HR changes occurred before embryonic death. Such HRFs may be a distinctive phenomenon of hypoxia. However, whether distinctive HRFs exist in hypoxia should be assessed in further research.
\end{abstract}

Keywords: chick embryo, heart rate fluctuations, autonomic nervous system activity, spectrum analysis

\section{Introduction}

Successful fetal development is significantly important for all animals (e.g., [1] [2]). Chick embryos develop externally by supplying suitable temperature and oxygen, which provides multiple advantages for studying fetal development, such as the ability to measure embryonic physiological parameters that are not influenced by maternal health conditions and to assess responses to changes in incubation environment (e.g., temperature, humidity, and oxygen concentration) (e.g., [3] [6]). Fertile chicken eggs are easy to prepare compared with pregnant rats/mice. In addition, the development of heart structures in chick embryos is similar to that in mammals, namely the tube structure develops into four chambers: two atria and two ventricles. Although the respiration of chick embryos changes from gas exchange through the egg shell to breathing with lungs during hatching, the measurement of cardiovascular parameters is possible even in periods of drastic respirational change, i.e., the prenatal period [7] [9]. Therefore, physiological parameters in chick embryos have been investigated to be a suitable and independent embryonic measurement model, particularly for cardiovascular systems, during the prenatal

\footnotetext{
* Corresponding author: moriya @ hakodate-ct.ac.jp

$\dagger$ Department of Production Systems Engineering, National Institute of Technology, Hakodate College.

Tokura-cho 14-1, Hakodate City, Hokkaido, Japan 042-8501

¥ Support Center for Engineering Education, National Institute of Technology, Hakodate College.

Tokura-cho 14-1, Hakodate City, Hokkaido, Japan 042-8501

$\S$ Department of Production Systems Engineering, National Institute of Technology, Hakodate College.

Tokura-cho 14-1, Hakodate City, Hokkaido, Japan 042-8501
}

and hatching periods. Cardiovascular performance has been investigated at each stage of chick embryo development. In mammals, it is impossible to measure embryonic heart rate (HR) during accouchement, which is the period that corresponds to emergence from the egg shell. However, in chick embryos, this period is difficult to study because of noise, which is attributed to embryonic body movements.

However, we developed an instantaneous HR (IHR) system to continuously measure HR of chick embryos before and during hatching [10] [14]. Although the normal developmental pattern of the chick embryonic HR has been previously elucidated, a comparison with abnormal development was required to verify whether the pattern was actually normal [10] [15]. In this study, we compared HR fluctuations (HRFs) (i.e., HR variability and HR irregularity) and gradient of IHR spectra between successfully hatched prenatal chick embryos and unhatched prenatal chick embryos.

\section{Materials and Methods}

2.1 Chick embryos and their incubation Fertile eggs of broiler chickens were purchased from a local hatchery. The first day of incubation was designated as day 0 , with chick embryos generally being hatched by day 21 . The eggs were incubated in a forced draft incubator at $38^{\circ} \mathrm{C} \pm$ $2^{\circ} \mathrm{C}, 50 \%-60 \%$ relative humidity, and hourly rotation until day 16 [16] [17].

2.2 Continuous measurements of IHR On day 17, electrodes were implanted between the egg shell and chorioallantoic membrane, and electrocardiogram (ECG) measurements were continuously performed from day 18 until hatching. Three electrodes were forming a triangle 
around the egg. Two sites were on the underside of the egg between the air cell and egg equator and made up the bottom of a triangle when the egg was placed in the horizontal position. The third site was marked on the top of the egg. After ECG waves with all combinations of electrodes (i.e., two electrodes were signal-detecting-electrodes, the other was for the ground electrode) were checked, the better combination that obtained the high signal to noise $(\mathrm{S} / \mathrm{N})$ ratio of electrodes were determined for the measurements. When the $\mathrm{S} / \mathrm{N}$ ratio decreased due to the movements of the embryos, the ground electrode was changed to the others. The eggs were not touched, and the incubator doors were not opened during the experiment. IHR was determined from adjacent $\mathrm{R}$ waves of ECGs, which were measured using the three-electrode method after analog and digital signal processing [10] [15].

\subsection{Spectrum analysis and index of spectrum structure}

Power spectrum analysis of IHR was performed using discrete Fourier transform after Hamming window was applied [18] [19]. To reduce the noise effect (incorrect IHR), each 10-min interval was divided into 100-s segments and spectrum components of each segment were averaged. Thus, the frequency resolution is $0.01 \mathrm{~Hz}$ and maximum frequency depends on a mean HR (e.g. if mean HR is $360 \mathrm{bpm}$, that is 6 $\mathrm{Hz}$, meaningful maximum frequency is $3 \mathrm{~Hz}$ ) [20].

A gradient of spectrum is used for the index of characteristic of spectrum structure. Usually, because the spectrum density $(\mathrm{P})$ is inversely to the frequency (f) on the log-log plot; therefore, the value of $\alpha$ is the gradient of the spectrum. The gradient $\alpha$ is determined by least square method (i.e., linear approximation) with steepest descent.

2.4 Indices of the sympathetic and parasympathetic nervous system activity In chick embryos, low frequency (LF) and high frequency (HF) components are 0.04$0.15 \mathrm{~Hz}$ and $0.1-1.0 \mathrm{~Hz}$, respectively [18]. HF component is the index of parasympathetic nervous system activity and the $\mathrm{LF} / \mathrm{HF}$ ratio is the index of sympathetic nervous system activity. Normalized HF components (\%HF), represents the ratio of $\mathrm{HF}$ component to $\mathrm{HF}+\mathrm{LF}$ and is used as an index of parasympathetic nervous system activity and reduces influence from very low frequency component. Each component was calculated by a numerical integration [20] [21].

\section{Results and Discussion}

In this study, we successfully measured IHR of prenatal chick embryos continuously until hatching. Figure 1 shows the developmental pattern of HR from day 18 until hatching. To comparatively assess long-term changes, MHR was used for the plot because the broad fluctuation in IHR obscures the developmental pattern of baseline HR. Respiration in chick embryos converts from gas exchange through the egg shell to breathing with lungs during hatching. Chicks pip the membrane of the egg ' $\mathrm{s}$ air cell to begin normal respiration with lungs on day 19 . This behavior is called internal pipping (IP), which indicates the preparation for hatching. Furthermore, chicks pip the eggshell on day 20 to obtain more oxygen, which is needed for the physiological devel-

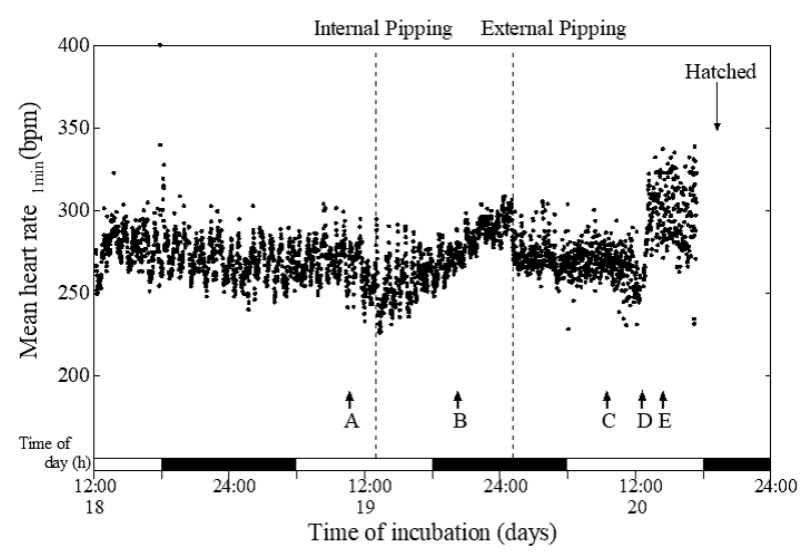

Figure 1: Developmental pattern of HR in a successfully hatched embryo. Black dots are MHR per minute. The horizontal axis indicates the time of incubation, and white and black boxes indicate the day time (6:00 to 18:00) and night time (18:00 to 6:00), respectively. The vertical dotted lines show IP and EP. A-E correlate with the spectra in Fig. 2.

opment required for hatching. This pipping is called external pipping (EP) and indicates that the chick will emerge from the shell within a day [7]. After that, respiration completely shifts to lung breathing. Baseline HR was approximately $280 \mathrm{bpm}$ on day 18 and gradually decreased until IP was detected around 13:00 on day 19. Subsequently, baseline HR increased until around 1:00 on day 20 when EP was detected. Baseline HR quickly decreased after EP and remained steady for approximately $12 \mathrm{~h}$. HR drastically accelerated around 12:00 on day 20 , at which point the chick successfully hatched. This trend of HR changes that accompany embryonic physiological milestones (e.g., IP, EP, and emergence from the shell) was commonly observed in normal/successfully hatched embryos $(\mathrm{N}=6)$.

HRF, that is, spontaneous, long-term, and brief HR accelerations and decelerations, often occur during development. During pre-IP on day 19, HR decelerations were dominant (Fig. 2A). HR accelerations also occurred after IP (Fig. 2B). The value of $\alpha$ is the gradient of the spectrum that increased after IP.

After EP, HR was relatively low and stable, likely because of the need for rest and oxygen intake in preparation for the subsequent hatching. Cyclic HR changes (respiratory arrhythmia) indicated by the peak of $0.6 \mathrm{~Hz}$ in the spectrum were observed (Fig. 2C). In chick embryos, low frequency (LF) and high frequency (HF) components are $0.04-0.15 \mathrm{~Hz}$ and $0.1-1.0 \mathrm{~Hz}$, respectively. After the chick started emerging from the egg shell, large cyclic HR accelerations were dominant (Fig. 2D, E). Furthermore, $\alpha$ increased to the maximum level toward the end of the hatching period. These patterns of IHR and gradient changes were commonly observed in normally hatched chicks.

Figure 3 shows the developmental pattern of MHR in an unhatched embryo. Baseline HR increased until 6:00 on day 19 , and HR did not vary compared with that of a normally developed embryo (Fig. 1), particularly from 24:00 on day 18. Thereafter, HR abruptly decreased, at which point the 


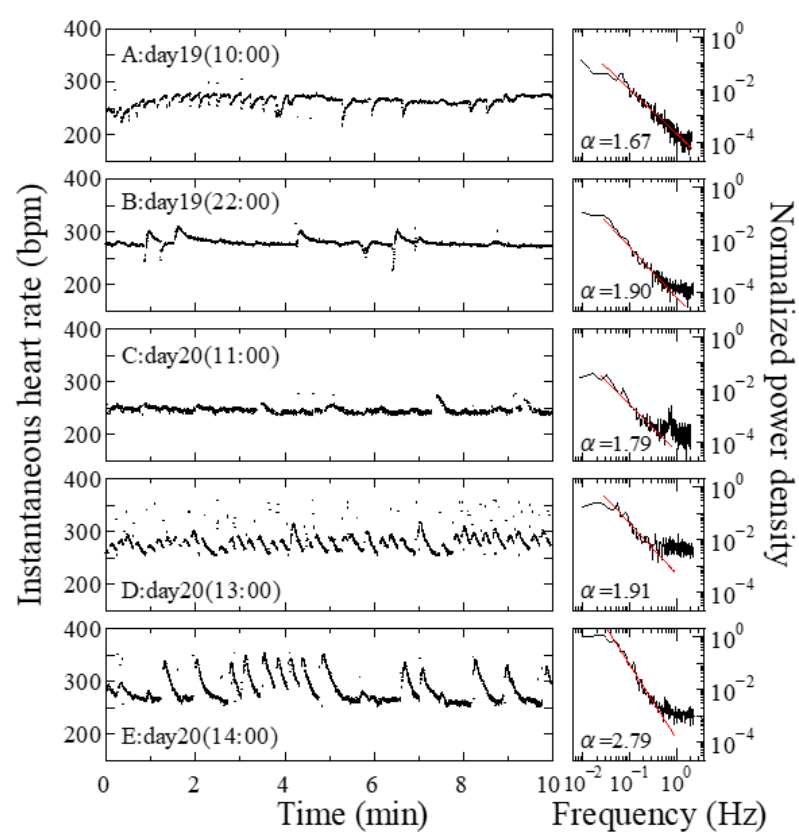

Figure 2: Normalized spectrum power density of 10-min IHR recordings at each developmental stage. A. Pre-IP HR. B. Post-IP HR. C. Steady post-EP HR. D. Continuous post-EP HRF. E. Intermittent post-EP HRF. A-E correlate with Fig. 1. Black dots are heart beats (IHR), and the red solid line is the gradient $\alpha$ of the spectrum calculated by the least square method.

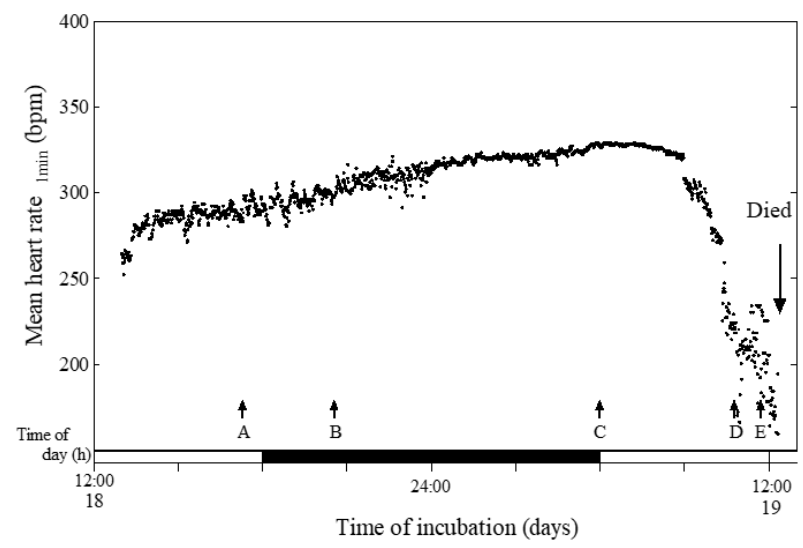

Figure 3: Developmental pattern of MHR in an unhatched embryo. Black dots are MHR per minute. The horizontal axis indicates the time of incubation, and white and black boxes indicate the day time (6:00 to 18:00) and night time (18:00 to 6:00), respectively. A-E correlate with the spectra in Fig. 4.

embryo died. This pattern of HR changes was distinctly different from a normally developed embryo. This embryo did not exhibit IP, which correlates with the absence of the pattern of HR deceleration and acceleration.

At 17:00 on Day 18, HR decelerations of approximately $50 \mathrm{bpm}$ were observed, similar to the IHR patterns of a normally developed embryo (Fig. 4A). However, the spectrum gradient ( $\alpha=1.49$ ) was lower than that of the normally developed embryo $(\alpha=1.67)$. At around $20: 00$ on day 18 ,

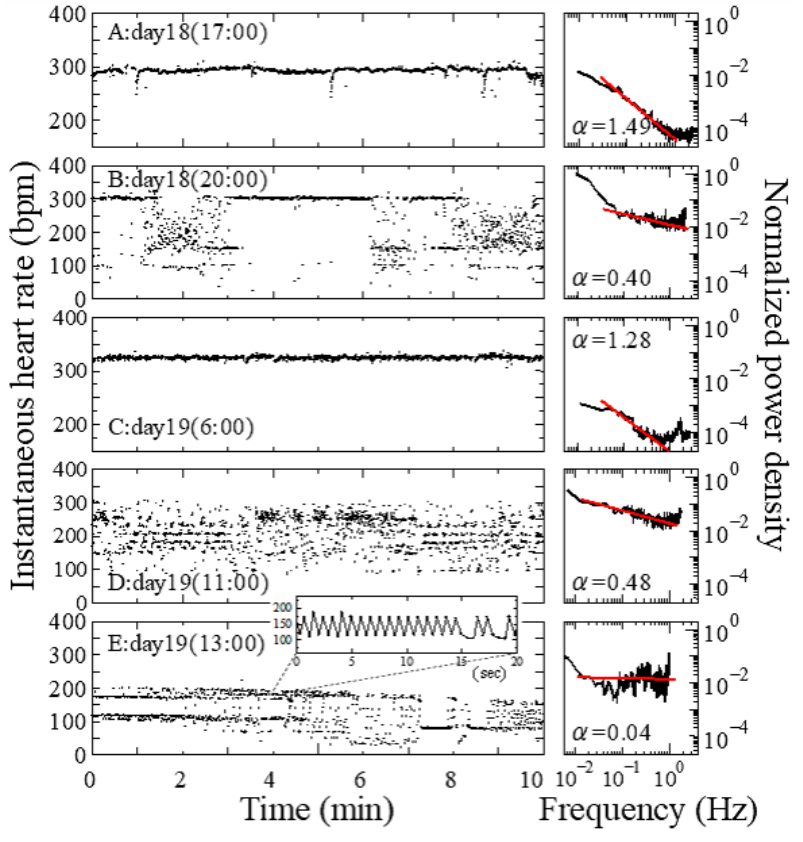

Figure 4: Ten-min IHR recordings and its spectrum density. A. Seemingly normal HR. B. Intermittent HR deceleration. C. Non-cyclic HR. D. Broad HRF. E. Just before death. Inset panel: $20 \mathrm{~s}$ excerpt of E. A-E correlate with Fig. 3. Black dots are heart beats (IHR), and the red solid line is a gradient $\alpha$ of the spectrum calculated by the least square method.

distinct abnormal HR decelerations intermittently occurred, and $\alpha$ was 0.40 , a remarkably abnormal value (Fig. 4B). These results indicate abnormal biological and/or physiological function(s) of this embryo. At 6:00 on day 19, the IHR pattern at first appeared normal, although baseline HR was higher. IHR was not cyclic, and $\alpha$ was low (1.28), implying that HRF did not spontaneously occur (Fig. 4C). After $5 \mathrm{~h}$, IHR randomly and broadly accelerated and decelerated (Fig. 4D). These data indicate that the embryo was unable to autonomously control heartbeat. Remarkably, just before death, HR alternated between low HR and high HR every beat (Fig. 4E). This HRF and the extremely low $\alpha$ value of 0.04 indicated that this embryo was near death. Because this embryo did not perform IP, adequate oxygen was not available to maintain a physiological function. Therefore, this characteristic cyclic HRF could be a distinctive physiological signal of hypoxia.

MHR per minute, autonomic nervous function indices (LF/HF and \% HF, averaged every $30 \mathrm{~min}$ ), and $\alpha$ (averaged every $30 \mathrm{~min}$ ) for a normally developed embryo (Fig. 5). Developmental patterns of baseline HR and $\alpha$ were similar to those of the embryo shown in Fig. 1. The sympathetic nervous function augmented at IP and before EP and was then dominant until hatching.

Figure 6 shows the developmental patterns of MHR, indices of autonomic nervous function, and $\alpha$ in an unhatched embryo, which is different from the one shown in Fig. 3. From approximately 18:00 on day 18 to about 1:00 on day 19, low-level and unstable HR, parasympathetic nervous function augmentation, and low $\alpha$ were observed. Then, 


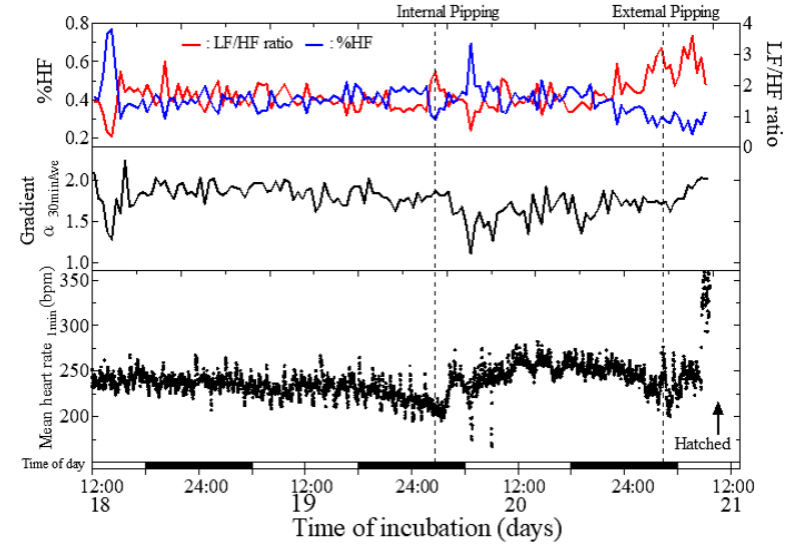

Figure 5: MHR, LF/HF ratio, \%HF, and the spectrum gradient $\alpha$ in a normally hatched embryo.

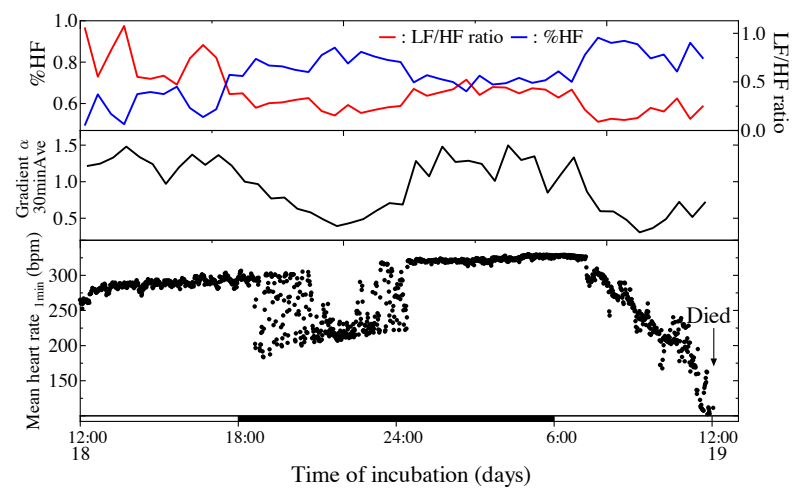

Figure 6: MHR, LF/HF ratio, \%HF, and $\alpha$ in an unhatched embryo.

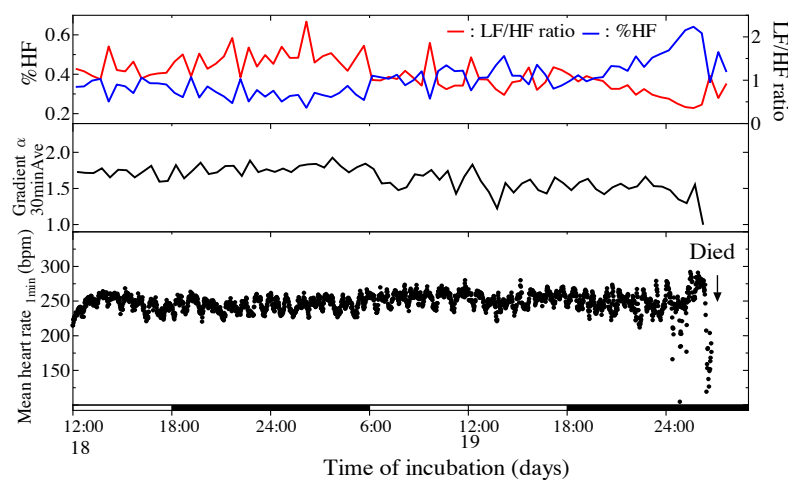

Figure 7: Unsuccessfully hatched embryo. HR and cyclic HRF (cyclic period of 40-60 min is defined as ultradian rhythm) as observed in normally developed embryos.

from 7:00 on day 19, MHR decreased, and the embryo died at 12:00 on day 19. Accompanying the decrease in MHR, parasympathetic nervous function was dominant and $\alpha$ decreased, indicating that antagonistic autonomic nervous function was not balanced.

An additional example of an unsuccessfully hatched embryo is shown in Fig. 7. HRF was cyclic within a period of approximately $1 \mathrm{~h}$ and did not decrease until before embryonic death. Previous studies have defined this fluctuation as an ultradian rhythm, which is observed in the late stages of normal embryonic development. Therefore, changes in MHR appeared to be normal development, although baseline HR did not decrease. However, $\alpha$ gradually decreased from 6:00 on day 19 and \%HF already increased approximately $7 \mathrm{~h}$ before death. These data indicate that spectrum analysis (determining $\mathrm{LF} / \mathrm{HF}$ ratio, $\% \mathrm{HF}$, and $\alpha$ ) is an applicable method to speculate an abnormality or dyscrasia of the embryonic physical development, which is not observed in a time-series analysis of HR.

\section{Conclusions}

The developmental pattern of long-term MHR, IHR fluctuation, and autonomic nervous system function were investigated in hatched and unhatched chicks using spectrum analysis. The characteristic developmental pattern of MHR that accompanies IP and EP, and HRF and its spectrum structure (i.e., $\alpha$ ) were revealed. Physiological parameters in three unhatched embryos, which failed to perform IP, were also measured. MHR and the HRF pattern were distinctly different from those of normally developed embryos. Antagonistic activities of sympathetic and parasympathetic nervous functions were unbalanced. Abnormal embryonic development is easily speculated by monitoring time-series ECG or IHR data.

However, the MHR pattern in one of the embryos appeared to maintain a normal fluctuation pattern, which could make speculation of abnormal physiological development by HR monitoring difficult. However, distinctive activity of autonomic nervous system and/or gradient of the spectrum were also observed. Monitoring these indices is effective for speculating abnormal embryonic development. However, more experimental data in both normal and abnormal embryos are required. Interestingly, characteristic HRF occurred just before embryonic death. Further studies with varying oxygen concentrations will verify whether this HRF is a distinctive signal of hypoxia. Moreover, whether distinctive HRF or distinctive HR spectra are indicative of specific diseases should be assessed in future studies.

\section{Acknowledgment}

A part of this study was supported by a Grant-in-Aid for Scientific Research (K.M. JP16K06397) from the Japan Society for the Promotion of Science.

\section{Additional statements}

This research was approved by the Life Ethics Committee of NIT, Hakodate College.

\section{References}

[1] Y. Sorokin, L. J. Dierker, S. K. Pillay, I. E. Zador, M. L. Schreiner and M. G. Rosen, "The association between fetal heart rate patterns and fetal movements in pregnancies between 20 and 30 week's gestation", American Journal of Obstet. Gynecol., 143, pp.243-249, 1982. 
[2] T. Wheeler and A. Murrills, "Patterns of fetal heart rate during normal pregnacy”, Br. J. Obstet. Gynaecol., 85, pp.18-27, 1978.

[3] H. Tazawa and H. Rahn, "Tolerance of chick embryos to low temperatures in reference to the heart rate", Comp. Biochem. Physiol, 85A, pp.531-534, 1986.

[4] G. C. Whittow and H. Tazawa, "The early development of thermoregulation in birds", Physiol. Zool., 64, pp.13711390,1991

[5] H. Tazawa, H. Wakayama, J. S. Turner, and C. V. Paganelli, "Metabolic compensation for gradual cooling in developing chick embryos", Comp. Biochem. Physiol., 89A, pp.125129, 1988.

[6] Y. Chiba, A. H. Khandokker, M. Nobuta, K. Moriya, R. Akiyama and H. Tazawa, "Ontogeny of respiratory rhythms in perinatal chick embryos", Comp. Biochem. Physiol., 131A, pp.817-824, 2002.

[7] N. Wang, J. P. Butler and R. B. Banzett, "Gas exchange across avian eggshells oscillates in phase with heartbeat", J. Appl. Physiol., 69, pp.1546-1552, 1990.

[8] J. Höchel, R. Akiyama, T. Masuko, J. T. Pearson, M. Nichelmann and H. Tazawa, "Development of heart rate irregularities in chick embryos", Am. J. Physiol.: Heart Cir. Physiol., 275, pp.H527-H533, 1998.

[9] J. T. Pearson, M. A. Haque, P.-C. L. Hou and H. Tazawa, "Developmental patterns of $\mathrm{O} 2$ consumption, heart rate and O2 pulse in unturned eggs”, Respir. Physiol., 103, pp.83-87, 1996.

[10] K. Moriya, J. T. Pearson, W. W. Burggren, A. R. Armos and H. Tazawa, "Continuous measurement of instantaneous heart rate and It' $\mathrm{s}$ fluctuations before and after hatching in chickens", Journal of Experimental Biology, 203, pp.895903, 2000.

[11] R. Akiyama, A. Matsuhisa, J. T. Pearson and H. Tazawa, "Long-term measurement of heart rate in chicken eggs", Comp. Biochem. Physiol.”, 124, pp.485-492, 1999.

[12] R. Akiyama, H. Ono, J. Höchel, J. T. Pearson and H. Tazawa, "Noninvasive determination of instantaneous heart rate in developing avian embryos by means of acoustocardiogram", Med. \& Biol. Eng. \& Comput., 35, pp.323-327, 1997.

[13] H. Tazawa, T. Hiraguchi, O. Kuroda, S. G. Tullett and O. C. Deeming, "Embryonic heart rate during development of domesticated birds", Physiol. Zool. 64, pp.1002-1022, 1991.

[14] H. Tazawa, H. Mitsubayashi, M. Hirata, J. Höchel and J. T. Pearson, "Cardiac rhythms in chick embryos during hatching”, Comp. Biochem. Physiol., 124, pp.513-523, 1999.

[15] K. Moriya, J. Höchel, J. T. Pearson and H. Tazawa, "Cardiac rhythms in developing. chicks. Comparative Biochemistry and Physiology", 124, pp.463-470, 1999.

[16] H. Tazawa, "Adverse effect of failure to turn the avain egg on embryo oxygen exchange”, Respir. Physiol., 41, 137-142, 1980.

[17] D. A. T. New, "A critical period for the turning of hens' eggs”, J. Embryol. Exp. Morph., 5, pp.293-299, 1957.
[18] S. Akselrod, D. Gordon, F. A. Ubel, D. C. Shannon, A. C. Barger and R. J. Cohen, "Power spectrum analysis of heart rate fluctuation: a quantitative probe of beat-to-beat cardiovascular control", Science, 213, pp.220-222, 1981.

[19] F.J. Harris, "On the use of windows for harmonic analysis with the discrete Fourier transform", Proceedings of the IEEE, 66, pp.51-83, 1978.

[20] K. Moriya, Y. Chiba, H. Yoneta, R. Akiyama and H. Tazawa, "Simultaneous measurement of instantaneous heart rate and breathing activity in newly hatched chicks", Br. Poult. Sci., Vol.44, No.5, pp.761-766, 2003.

[21] K. Moriya, I. Kurimoto, N. Ezaki and M. Nakagawa, "Influences of listening to music in study break on brain activity and parasympathetic nervous system activity", Journal of Institute of Industrial Applications engineers, Vol.6, No.1, pp.34-38, 2018.

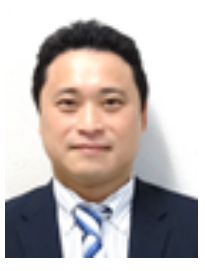

Kenji Moriya(Member) was born in Hokkaido, Japan, 1975. He received a Ph.D. degree in engineers from Muroran Institute of Technology in 2001, and is presently a Professor at National Institute of Technology, Hakodate College since 2016. He has worked on bio-measurement engineering and its signal processing. He is a member of IIAE, BMFSA, JSWE, JSEE and IEICE.

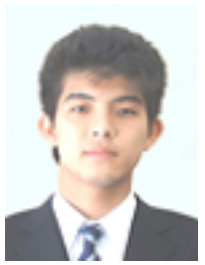

Yuya Chiba (Non-member) received an Associated degree in electrical and electronics engineering from National Institute of Technology, Hakodate College in 2012 and is presently a technical officer at NIT, Hakodate College.

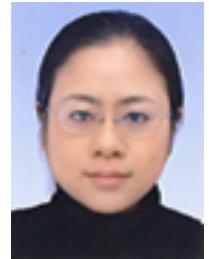

Yoshiko Maruyama (Non-member) received a Ph.D. degree in engineers from Kyoto Sangyo Univ. in 2013, and is presently an Assistant professor at National Institute of Technology, Hakodate College since 2016. She has worked on electrophysiology and bio-measurement engineering. 